\title{
Wopfner, Gabriele
}

\section{Zeichnungen als Schlüssel zu kindlichen Vorstellungen von Geschlechterbeziehungen}

Rendtorff, Barbara [Hrsg.]; Prengel, Annedore [Hrsg.]: Kinder und ihr Geschlecht. Opladen u.a. : Verlag Barbara Budrich 2008, S. 163-176. - (Jahrbuch Frauen- und Geschlechterforschung in der Erziehungswissenschaft; 4)

Quellenangabe/ Reference:

Wopfner, Gabriele: Zeichnungen als Schlüssel zu kindlichen Vorstellungen von

Geschlechterbeziehungen - In: Rendtorff, Barbara [Hrsg.]; Prengel, Annedore [Hrsg.]: Kinder und ihr Geschlecht. Opladen u.a. : Verlag Barbara Budrich 2008, S. 163-176 - URN:

urn:nbn:de:0111-opus-82359 - DOI: 10.25656/01:8235

https://nbn-resolving.org/urn:nbn:de:0111-opus-82359

https://doi.org/10.25656/01:8235

in Kooperation mit / in cooperation with:

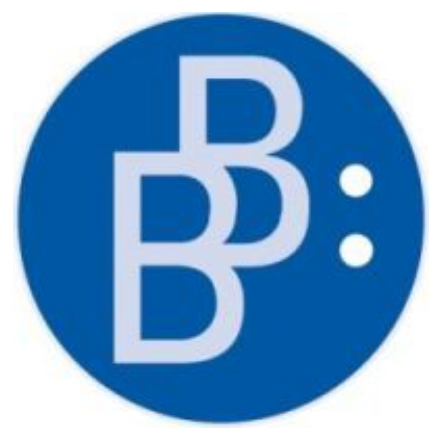

https://www.budrich.de

\section{Nutzungsbedingungen}

Gewährt wird ein nicht exklusives, nicht übertragbares, persönliches und beschränktes Recht auf Nutzung dieses Dokuments. Dieses Dokument ist ausschließlich für den persönlichen, nicht-kommerziellen Gebrauch bestimmt. Die Nutzung stellt keine Übertragung des Eigentumsrechts an diesem Dokument dar und gilt vorbehaltlich der folgenden Einschränkungen: Auf sämtlichen Kopien dieses Dokuments müssen alle Urheberrechtshinweise und sonstigen Hinweise auf gesetzlichen Schutz beibehalten werden. Sie dürfen dieses Dokument nicht in irgendeiner Weise abändern, noch dürfen Sie dieses Dokument für öffentliche oder kommerzielle Zwecke vervielfältigen, öffentlich ausstellen, aufführen, vertreiben oder anderweitig nutzen.

Mit der Verwendung dieses Dokuments erkennen Sie die Nutzungsbedingungen an.

\section{Terms of use}

We grant a non-exclusive, non-transferable, individual and limited right to using this document.

This document is solely intended for your personal, non-commercial use. Use of this document does not include any transfer of property rights and it is conditional to the following limitations: All of the copies of this documents must retain all copyright information and other information regarding legal protection. You are not allowed to alter this document in any way, to copy it for public or commercial purposes, to exhibit the document in public, to perform, distribute or otherwise use the document in public.

By using this particular document, you accept the above-stated conditions of use.

\section{Kontakt / Contact:}

peDOcs

DIPF | Leibniz-Institut für Bildungsforschung und Bildungsinformation Informationszentrum (IZ) Bildung

E-Mail:pedocs@dipf.de

Internet: www.pedocs.de

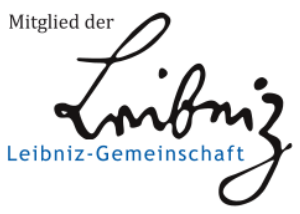


Kinder und ihr Geschlecht 
Jahrbuch der

Frauen- und Geschlechterforschung

in der Erziehungswissenschaft

herausgegeben von

Rita Casale

Barbara Rendtorff

Sabine Andresen

Vera Moser

Annedore Prengel

Beirat

Birgit Althans, Berlin

Eva Borst, Mainz

Eva Breitenbach, Osnabrück

Bettina Dausien, Bielefeld/München

Isabell Diehm, Bielefeld

Hannelore Faulstich-Wieland, Hamburg

Edgar Forster, Salzburg

Edith Glaser, Dortmund

Carola Iller, Heidelberg

Andrea Liesner, Hamburg

Susanne Maurer, Marburg

Inga Pinhard, Frankfurt

Folge 4/2008 
Barbara Rendtorff

Annedore Prengel (Hrsg.)

Kinder und ihr Geschlecht

Verlag Barbara Budrich

Opladen \& Farmington Hills 2008 
Bibliografische Informationen der Deutschen Nationalbibliothek

Die Deutsche Nationalbibliothek verzeichnet diese Publikation in der Deutschen

Nationalbibliografie; detaillierte bibliografische Daten sind im Internet über

http://dnb.d-nb.de abrufbar.

Gedruckt auf säurefreiem und alterungsbeständigem Papier.

Die Deutsche Bibliothek - CIP-Einheitsaufnahme

Ein Titeldatensatz für die Publikation ist bei Der Deutschen Bibliothek erhältlich.

Alle Rechte vorbehalten.

(C) 2008 Verlag Barbara Budrich, Opladen

www.budrich-verlag.de

\section{ISBN 978-3-86649-181-6}

Das Werk einschließlich aller seiner Teile ist urheberrechtlich geschützt. Jede Verwertung außerhalb der engen Grenzen des Urheberrechtsgesetzes ist ohne Zustimmung des Verlages unzulässig und strafbar. Das gilt insbesondere für Vervielfältigungen, Übersetzungen, Mikroverfilmungen und die Einspeicherung und Verarbeitung in elektronischen Systemen.

Umschlaggestaltung: disegno visuelle kommunikation, Wuppertal - www.disenjo.de Satz: R+S Beate Glaubitz, Leverkusen

Druck: paper\&tinta, Warschau

Printed in Europe 


\section{Inhalt}

\section{Zur Einführung}

Annedore Prengel/Barbara Rendtorff

Kinder und ihr Geschlecht - Vielschichtige Prozesse und punktuelle

Erkenntnisse

\section{Gastbeitrag}

Caryl Rivers/Rose Barnett

The difference myth. We shouldn't believe the increasingly popular claims that boys and girls think differently, learn differently, and need to be treated differently

\section{Beiträge}

Sabine Andresen

Kinder und soziale Ungleichheit. Ergebnisse der Kindheitsforschung zu dem Zusammenhang von Klasse und Geschlecht .....

Hans Peter Kuhn

Geschlechterverhältnisse in der Schule: Sind die Jungen jetzt benachteiligt? Eine Sichtung empirischer Studien

Ulrike Schmauch

Gleichgeschlechtliche Orientierungen von Mädchen und Jungen -

Eine Herausforderung an die Pädagogik

Anja Zeiske/Alexandra Klein/Hans Oswald

Die Lust beim ersten Mal: Jugendliche und die Bewertung ihres ersten Geschlechtsverkehrs 


\section{Aus der Forschung}

Frank Hellmich/Sylvia Jahnke-Klein

Selbstbezogene Kognitionen und Interessen von Mädchen und Jungen im Mathematikunterricht der Grundschule

Ruth Michalek/Thomas Fuhr

Hegemonialität und Akzeptanz von Abweichung in Jungengruppen.

Empirische Studien zum Umgang mit Opposition

Stephan Mücke/Agi Schründer-Lenzen

Zur Parallelität der Schulleistungsentwicklung von Jungen und

Mädchen im Verlauf der Grundschule

\section{Work in Progress}

\section{Christine Rabl/Elisabeth Sattler}

Anderssein - Anderswerden. Zur Revision der Relationierung von

Kindheit und Geschlecht aus differenztheoretischer Sicht

Gabriele Wopfner

Zeichnungen als Schlüssel zu kindlichen Vorstellungen von

Geschlechterbeziehungen

Jutta Wiesemann

Schulischer Erfolg ist weiblich: Welche schulische Praxis verbirgt sich hinter den Zahlen der Schulstatistik?

Dagmar Kasüschke

Geschlechtsbezogene Wissenskonzepte von Kindern

unter sechs Jahren - ein Problemaufriss

\section{Rezensionen}

Marita Kampshoff

Rezension zu: Emma Renold (2005): Girls, boys and junior sexualities:

exploring children's gender and sexual relations in the primary school .. 203

Eva Borst

Rezension zu: Helga Bilden/Bettina Dausien (Hg. 2006): Sozialisation und Geschlecht. Theoretische und methodologische Aspekte 
Inhalt

Barbara Scholand

Rezension zu: Suthues, Bettina (2006): Umstrittene Zugehörigkeiten.

Positionierungen von Mädchen in einem Jugendverband.

Dietlind Fischer

Rezension zu: Doris Lemmermöhle et al. (2006): Passagen und

Passantinnen. Biographisches Lernen junger Frauen. Eine

Längsschnittstudie

Über die AutorInnen dieses Bandes

Zum Jahrbuch Frauen- und Geschlechterforschung in der Erziehungswissenschaft

Ankündigung der nächsten Bände 226

Abonnements- und Bestellvordruck 


\section{Zeichnungen als Schlüssel zu kindlichen Vorstellungen von Geschlechterbeziehungen}

\section{Gabriele Wopfner}

Das Dissertationsprojekt „Kindliche Vorstellungen von Geschlechterbeziehungen“ zielt auf die intrapsychische Auseinandersetzung von Mädchen und Jungen mit „Geschlecht" und „Geschlechtszugehörigkeit" und untersucht die kindlichen Vorstellungen von Beziehungen zu den Anderen am Beginn der Adoleszenz in einer Studie mit 90 Kindern an drei österreichischen Schulen.

Die Ergebnisdarstellung aus 22 der dokumentarisch interpretierten Kinderzeichnungen zeigt alters- und geschlechtsspezifische Unterschiede in der Zusammensetzung der gezeichneten Peergruppe, der Darstellung der Personen und der situativen Rahmung. Zwei Bildinterpretationen thematisieren exemplarisch die kindliche Auseinandersetzung mit ihrem „Zur-Frau/Zum-Mann-Werden“ und bringen die Ergebnisse in Verbindung mit dem intersubjektiven Paradigma der Relationalen Psychoanalyse.

\section{Drawings as Keys to Childlike Images of Gender Relationships}

The project of the thesis „On Childlike Perceptions of Gender Relationship“ examines the intra-psychical analysis of girls and boys regarding gender and gender identity as well as the childlike notion of relations to the 'others' at the beginning of adolescence in a study with 90 children from three Austrian schools.

The presentation of the findings of 22 of the children's drawings, which were interpreted by means of the documentary method, reveals sex and age specific differences in the composition of the depicted peer group, the presentation of individuals and in the framework of the situation. Two exemplary drawing interpretations thematically demonstrate the childlike notions as to how children deal with developing their gender identity and connect these results with the intersubjective paradigm of relational psychoanalysis.

Das im Folgenden vorgestellte Dissertationsprojekt „Kindliche Vorstellungen von Geschlechterbeziehungen“ zielt auf die intrapsychische Auseinandersetzung von Mädchen und Jungen mit „Geschlecht“ und „Geschlechtszugehörigkeit" und untersucht die kindlichen Vorstellungen von Beziehungen zu den Anderen am Beginn der Adoleszenz. 
Im Mittelpunkt des forschungsmethodischen Zugangs steht das kreative Gestalten - die Kinderzeichnung. Als primäre Auswertungsmethode wählte ich die dokumentarische Methode der Bildinterpretation. Sie fokussiert in ihrer nicht nur „erkennenden“ sondern ,sehenden“ Betrachtungsweise auf das atheoretische Wissen der Bildproduzent/inn/en (vgl. Imdahl 1996, Bohnsack 2007) und lässt damit die bewussten und unbewussten Vorstellungen von Geschlechterbeziehungen in den Blick kommen. Die anschließende komparative Analyse, die die Zeichnungen von Kindern aus verschiedensten schulischen Einzugsgebieten (Stadtschule, Landschule, Schule mit sehr hohem Ausländer/innenanteil) bearbeitet, weitet den Blick auf die im Forschungsdesign angelegte Frage nach den Typiken und eventuellen bildungs- und milieuspezifischen Unterschieden. Durch die ergänzende, in diesem Text nicht zu behandelnde, Analyse von Kinderaufsätzen, Einzelinterviews und Gruppendiskussionen sollen die Bildinterpretationen - in einer Methodentriangulation - gestützt werden.

Der empirische Teil der Studie wurde im Schuljahr 2005/06 mit 90 Kindern an drei österreichischen Schulen durchgeführt. Die Basis bilden 90 Zeichnungen und Aufsätze, 12 Einzelinterviews und 9 Gruppendiskussionen.

Zielgruppe sind Kinder in der 6. Schulstufe. Sie sind im Alter von 11/12 Jahren und befinden sich in der Regel lebensgeschichtlich am Beginn der Adoleszenz. Durch die körperlichen Veränderungen, die bedingt durch die Akzeleration in diesem Alter teilweise schon verstärkt einsetzen, gewinnen gesellschaftliche Diskurse über Weiblichkeit und Männlichkeit auch bezogen auf sexuelle Orientierungen und soziale Normierungen an Bedeutung, die psychosexuelle Dynamik wird durch sie mitbeeinflusst.

Schule als „Identitätsbaustelle“ - dieser einem Zitat von Annamarie Jagose entlehnte Begriff begleitet als Metapher die Studie. Jagose verwendet den Begriff für queer und beschreibt „Identitätsbaustelle“ als ,ein(en) Ort beständigen Werdens“ $(2001,165)$. Da das „Zum-Geschlecht-Werden“ in der ausgehenden Kindheit Brüche und Inkohärenzen aufweisen kann und queer auch für alle Arten von Geschlechtsinszenierungen, die die Geschlechtergrenzen irritieren, verwendet wird, soll der Begriff „Baustelle“ ein Bild evozieren, dass die Auseinandersetzung mit (Geschlechts-)Identität nie wirklich abgeschlossen werden kann, der Prozess ein „beständiges Werden“ enthält. Schule mit ihren vielfältigen Beziehungsangeboten wird als ein Ort solcher Auseinandersetzungen betrachtet. Der Terminus „Baustelle“ verweist zudem auf ein de-/konstruktivistisches Verständnis von (Geschlechts-)Identität und öffnet über die Betonung der Prozesshaftigkeit des Ortes Schule den Blick auf Chancen und Herausforderungen pädagogischen Handelns. 
Das der Studie zugrunde liegende sozialisationstheoretische Konzept betrachtet das Kind als aktives, sich mit seiner Umwelt auseinandersetzendes und seine Biografie gestaltendes Subjekt (vgl. Hurrelmann 1999, Dausien 2006). In der alterstypischen Umbruchphase zum Jugendlichen sind die Kinder als „schöpferische Akteure ihrer eigenen Lebenswelt“ (Bründel/Hurrelmann 1996, S. 72) eingebunden in einen ständigen Prozess des Suchens und Tastens, des Ausprobierens von Spielräumen und Verhaltensmöglichkeiten. Es stellt sich ihnen verstärkt die Aufgabe, an der Entwicklung eines identitätsstiftenden Selbstbildes zu arbeiten. Die damit einhergehenden innerpsychischen Unsicherheiten, Brüche und Inkohärenzen legen eine zusätzliche, psychoanalytisch orientierte Betrachtungsweise nahe. Jessica Benjamins intersubjektives Paradigma $(1996,2002)$ regt dazu an, die kindlichen Vorstellungen von Interaktionen und Geschlechterbeziehungen auch aus dem Blickwinkel der Relationalen Psychoanalyse zu betrachten. Die Auswertung der erhobenen Daten (Zeichnungen, Interviews und Gruppendiskussionen) setzt auf der Ebene des Individuums an, geht aber in der Analyseeinstellung der dokumentarischen Methode darüber hinaus und zielt auf das Verstehen der habitualisierten Beziehungsvorstellungen der Kinder - individuell und kollektiv. Im Mittelpunkt des Forschungsinteresses steht damit der kindliche Habitus, der ,modus operandi“", der als Prozessstruktur der sozialisationsgeschichtlichen Entwicklung und dem habituellen Alltagshandeln der einzelnen Kinder zugrunde liegt. Die komparative Analyse sucht nach Gemeinsamkeiten in den Daten und fragt, ob ein kollektiver Habitus (in geschlechts- und/oder milieuspezifischer Hinsicht) erkennbar wird.

In diesem Artikel werde ich zuerst mein Forschungsdesign kurz beschreiben (1). Ich zeige auf, wie ich meine Daten - Zeichnungen, Aufsätzen und Interviews - erhoben habe und gehe dann auf die Auswertung der Bilder und forschungsrelevante Fragestellungen ein (2). Im letzten Teil dieses Beitrages stelle ich erste Ergebnisse meiner Arbeit dar (3).

\section{Zur Durchführung des Projekts}

Über Zeichnungen, Texte, Einzelinterviews und Gruppendiskussionen wurde den Kindern eine Vielfalt von Möglichkeiten gegeben, ihren bewussten und unbewussten Vorstellungen von Geschlechterbeziehungen Ausdruck zu verleihen. Das Bild generierende Verfahren stand am Beginn der Forschungseinheit, denn „Kinderzeichnungen (sind) ein Schlüssel zum Denken und Fühlen eines Kindes“" (Reiß 2000, S. 35). 


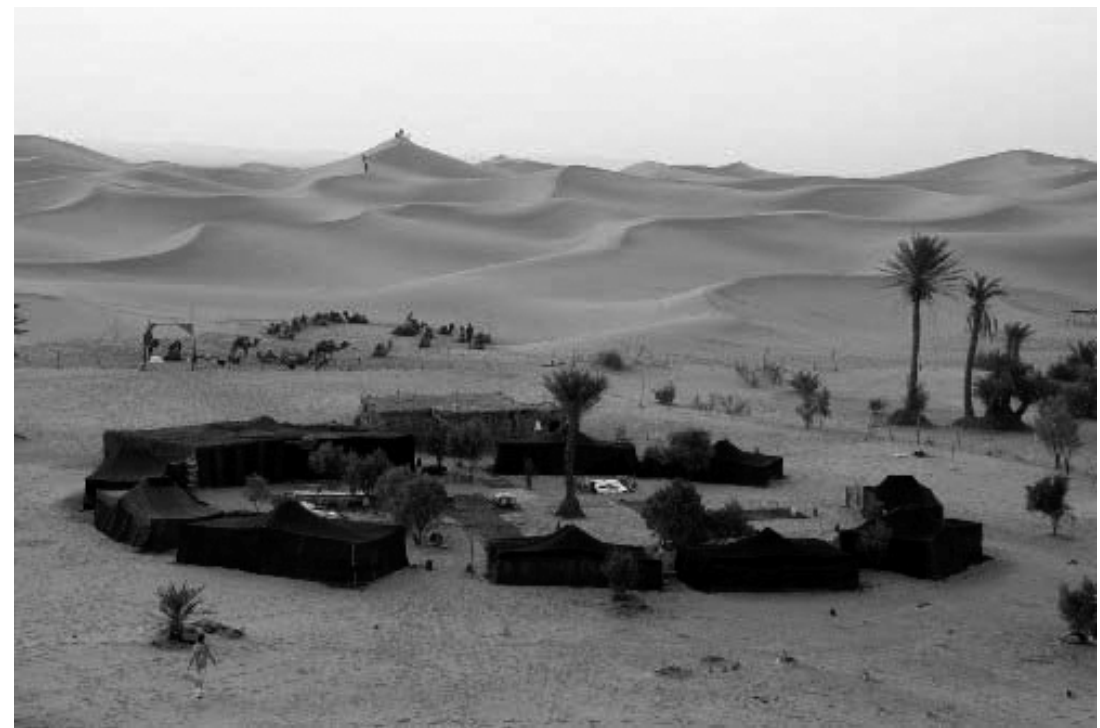

Abb. 1: Impulsbild „Wüste“

Als thematischer Bezugsrahmen diente die Vorstellung einer Wüstenexpedition. Die situative Rahmung ermöglichte es den Kindern, ihre individuellen Vorstellungen, Erfahrungen und Wünsche im Zusammenleben mit Gleichaltrigen einfließen zu lassen. Sie öffnet den Raum, um soziale Prägungen und internalisierte Muster sichtbar werden zu lassen. Als thematischer Impuls wurde den Kindern via Overhead ein Wüstenbild (Abb.1) gezeigt. Sie sollten sich vorstellen, mit 9 anderen Kindern vier Wochen in der Wüste zu verbringen. Eine Szene dieses Zusammenlebens sollte nun gezeichnet werden. In einer weiteren Unterrichtseinheit schrieben die Kinder einen Text über den Lageraufbau in der Wüste. In beiden Unterrichtsstunden wurden die Interaktionen der Mädchen und Jungen beobachtet, anhand des Sitzplans grafisch nachvollzogen und dokumentiert. Auf Basis von Bohnsacks Kriterium der maximalen Kontraste (1989, S. 18) für die Konturierung von Typiken wurden nach einer ersten Sichtung des gesammelten Materials vier bis sechs unterschiedliche Zeichnungen pro Klasse ausgewählt. In den anschließenden Einzelgesprächen sollten die Kinder ihre Bilder erklären, Auskunft geben über die Stimmungen der handelnden Personen und beschreiben, wie die Szene weitergehen könnte.

Für die Darstellung erster Ergebnisse aus meiner Untersuchung habe ich 22 Zeichnungen einer 6. Schulstufe an einem städtischen Gymnasium im 
Osten Österreichs ausgewählt. Informationen aus den Aufsätzen und Einzelinterviews fließen nur punktuell ein.

\section{Der Blick auf die Bilder und ihre dokumentarische Interpretation}

In einem ersten Schritt werden die Zeichnungen kurz beschrieben, um einen Eindruck von den gestalterischen Produkten der Kinder im Alter von 11 bis Ende 12 Jahren zu vermitteln. Im Anschluss werden erste Antwortmöglichkeiten auf Erkenntnis leitende Fragen des Dissertationsprojektes dargestellt. Dazu werden zur vergleichenden Betrachtung der Bilder, die auf der (vor-)ikonografischen Ebene bleibt und sich an den Phänomenen orientiert, auch zwei Bildinterpretationen herangezogen. Über die dokumentarische Bildinterpretation wird versucht, die Oberflächenebene der ikonografischen Bedeutung zu transzendieren, um Einblick zu erhalten in die Perspektive der abbildenden Bildproduzent/inn/en, ihre soziale Bezogenheit und eventuelle innerpsychischen Prozesse. Zentral für die dokumentarische Methode ist die methodologische Differenzierung von immanentem und dokumentarischem Sinngehalt. Für die Bildinterpretation ist dies der Wechsel der Analyseeinstellung von der Ikonografie (WAS gezeichnet wird) zur Ikonologie (WIE gezeichnet wird). Die Differenzierung der verschiedenen Sinnebenen verlangt eine klare Strukturierung der Interpretationsschritte - die formulierende und die reflektierende Interpretation. Ziel der dokumentarischen Methode ist der Zugang zum konjunktiven Erfahrungsraum der Bild-Produzent/inn/en, dessen zentrales Element der individuelle oder kollektive Habitus darstellt und die Handlungspraxis der Kinder strukturiert. Für den Wechsel der Analyseeinstellung vom WAS zum WIE ist im Rahmen der reflektierenden Interpretation die Rekonstruktion der Formalstruktur (Planimetrie) des Bildes wesentlich. Die Planimetrie erfasst den zugrunde liegenden Bildaufbau und gibt Aufschluss über mögliche Fokussierungen und Orientierungen der Bildproduzent/inn/en. Dieser formale Analyseschritt zielt auf eine Sinnebene, die uns über das textförmige Vor-Wissen - das ikonografische Wissen - nicht zugänglich ist (vgl. Bohnsack 2007, S. 166f.). Die „Planimetrische Gesamtstruktur“ ist damit Grundlage für das schon angesprochene „sehende Sehen“ und ermöglicht es, das Bild als ,ein nach immanenten Gesetzen konstruiertes und in seiner Eigensetzlichkeit evidentes System“ (Imdahl 1979, in: Bohnsack 2007, S. 167) zu betrachten. Die hier eingebrachten Bildinterpretationen 
wurden in einer dokumentarischen Interpretationsgruppe im Rahmen einer Forschungswerkstatt an der Universität Wien ${ }^{1}$ diskutiert.

Fragen an das Datenmaterial:

1) Wie bearbeiten die Kinder die in der Zeichnungsaufforderung implizit enthaltene Beziehungsthematik: Zeichne eine Szene, die du mit deinen Freundinnen und Freunden bei einer Wüstenexpedition erlebst ? Mit welcher Art von Gruppe - gleichgeschlechtlich oder heterogen zusammengestellt - möchten sie die vier Wochen in der Wüste erleben?

Diese Frage soll erste Hinweise geben auf die kindlichen Vorstellungen über die Zusammenstellung der Peergruppe, die affektive Bezogenheit zum gleichen wie zum anderen Geschlecht.

2) Wie stellen sich die Kinder zeichnerisch dar? Bringen die Bilder Vorstellungen vom eigenen Körper und dem der Anderen zum Ausdruck? Lassen sich in den Bildern Auseinandersetzungen mit den in der Pubertät beginnenden körperlichen Veränderungen finden?

Es ist mir bewusst, dass ich in diesem Beitrag - wie im ganzen Projekt - nur einen Ausschnitt der Thematik beleuchten kann, denn „(d)er Einzelblick, auf den angewiesen wir gerichtet sind, erweist sich als prinzipiell ungenügend, er verweist auf weiteres zu Erblickendes" (Graumann 1960, S. 178, in: Prengel 2000, S. 88). Auch möchte ich an dieser Stelle darauf hinweisen, dass die Methode der dokumentarischen Bildinterpretation noch sehr jung ist. Die methodologische Reflexion der zur Anwendung gelangenden Interpretationsverfahren steht erst am Anfang. Es liegen erst wenige Arbeiten vor, die zudem nur einen Fall bearbeiten. Hinsichtlich der komparativen Analyse gibt es noch keine Vorlagen.

\section{Erste Interpretationsergebnisse}

Bei der ersten Sichtung der Bilder drängt sich eine Unterteilung der Zeichnungen nach jüngeren und älteren Kindern stärker auf als eine geschlechtsspezifische Differenzierung. Es lassen sich bei den jüngeren Kindern Gemeinsamkeiten hinsichtlich der erkennbaren Themen und der Darstellungen der Figuren von Mädchen und Jungen feststellen. Ab dem Alter von ca. 12

1 Die Forschungswerkstatt „Höhere qualitative Forschungsmethoden“ fand im Sommersemester 2007 unter der Leitung von Frau Prof. Przyborski und Herrn Prof. Bohnsack statt. 
Jahren zeigen sich in dieser Klasse Unterschiede zwischen den Zeichnungen der Mädchen und der Jungen.

\subsection{Von „wildem Treiben“ und bedeutsamen Situationen}

Ein kriegerisches Thema herrscht in den Bildern der jüngeren Mädchen und Jungen - 11 bis 12 Jahren alt - vor. M1w $(11,5)^{2}$ und M2m $(11,7)$ zeichnen beide eine brutale Schlacht. Die Bilder sind auch in der Art ihrer Gestaltung sehr ähnlich. So zeichnet M1w wie die meisten Jungen fast ausschließlich Strichmännchen. Nur wenn sie einen besonderen Sachverhalt verdeutlicht z.B. wenn eine Person durch einen Messerstich in die Brust gestorben ist wird diese Person körperhaft dargestellt, der Oberkörper flächig gezeichnet. Hier deutet sich an, dass dieses „Flächig-werden“ ein besonderes Ausdrucksmittel der Mädchen und Jungen zu sein scheint.

In der Themenwahl lassen sich in dieser Klasse ab dem Alter von 12 Jahren Unterschiede zwischen Schülerinnen und Schülern erkennen. Die Jungen (12 bis 12,6 Jahre) zeichnen weiterhin abenteuerliche, zum Teil kriegerische Themen. Das „wilde Treiben“ spannt sich vom waghalsigen Bungeejumping am durchgetrennten Seil, dessen Ziel ein Grabstein mit der Aufschrift „Don't R.I.P.“ ist, zum Flug mit Kanonenkugeln und Sciencefiction ähnlichen Situationen mit motorisierten Reptilien. Die beiden ältesten (12,7 J.) zeichnen ein Fußballspiel.

Die älteren Mädchen zeichnen hingegen Situationen mit Bezug zu ihrem Alltag und betten diese großteils in den phantasierten Erlebnisraum der Wüstenexpedition ein. Die Art der Darstellung spiegelt den emotionalen Bedeutungsgehalt, den die gezeichneten Personen für die Bildproduzentin haben. Mimik, Gestik und Körperhaltung lassen affektive Bezogenheit erkennen. Es sind nicht immer die Freundinnen, die über die Ausdrucksproportion groß dargestellt werden und so Bedeutung erlangen. In einer Zeichnung befindet sich die „verhasste“ Gruppe, die das Klassenleben stark prägt, übergroß im Vordergrund, die Anderen - zu denen auch die Bildproduzentin gehört - sind draußen in den Dünen als Strichfiguren dargestellt.

2 Die Bilder und Texte der Kinder wurden anonymisiert: das M steht für den Phantasienamen der Schule „Marienberg“", w = weiblich, $m$ = männlich. Die Ziffer nach dem Großbuchstaben bezieht sich auf den Platz, den das Kind seinem Alter entsprechend in der Klasse einnimmt. Da das Alter ein wichtiges Datum darstellt, wird es in der Klammer präzisiert. M1w(11,5) ist also das jüngste Mädchen dieser Klasse, das im Alter von 11,5 Jahren an diesem Forschungsprojekt teilgenommen hat. 


\subsection{Der „Strich“ nimmt Formen an - vom Strichmännchen zum „Körper von Gewicht"6}

Auch wenn die Zeichenaufforderung auf 10 darzustellende Kinder zielte, unterscheidet sich der Blick auf die Gruppe in den Bildern. Ist es bei den jüngeren Kindern mindestens eine 10er Gruppe, so nimmt die Anzahl der Gruppenmitglieder in den Zeichnungen der älteren Kinder (ab 12,6 Jahren), bis auf zwei Ausnahmen, ab.

Die älteren Mädchen konzentrieren sich in ihren Darstellungen vermehrt auf Details einzelner Personen. Die gleichgeschlechtlichen Partnerinnen werden zunehmend größer und differenzierter dargestellt. Sie sind körperhafter gezeichnet und werden auch in ihrer Weiblichkeit abgebildet. Ist in den Zeichnungen der jüngeren Mädchen das Geschlecht noch als Symbol dargestellt, so sind in den späteren Zeichnungen die sekundären Geschlechtsmerkmale sichtbar. Die Personendarstellungen unterscheiden sich, der „Strich“ nimmt Formen an, wird körperhafter und runder. Die Brüste werden sichtbar. Die Assoziation „Körper von Gewicht“, wie Judith Butler (1995) ihr Buch nennt, drängt sich auf. Die Personen sind nicht mehr nur Identitätsträger/innen qua Symbol, sie werden als Individuen sichtbar und können voneinander unterschieden werden. Spielt bei den jüngeren Mädchen die Gruppe bzw. deren Darstellung noch eine große Rolle, so richtet sich der Blick der älteren Mädchen (ab 12,6) auf wenige Freundinnen. Die detailreiche Darstellung lässt ein Vertrautsein mit Eigenheiten erkennen, gleichzeitig geraten die Anderen mehr in den Hintergrund bzw. verschwinden sie aus dem Bild.

Die Darstellung von Körpern in den Zeichnungen der Jungen weist Parallelen auf. In ihren Zeichnungen finden wir durchweg Strichfiguren. Erst der älteste Junge (12,7 Jahre) zeichnet einige seiner Figuren körperhaft. Über breite Oberkörper und skizzenhaft gezeichnete Geschlechtsorgane deutet er Attribute von Männlichkeit an. Die Anzahl der Gruppenmitglieder bleibt bei den Jungen gleich, eine Reduktion der dargestellten Personen, wie bei den Mädchen, kann nicht festgestellt werden. Die beiden Jungen sind die Ausnahmen in der Bilderreihe der älteren Kinder.

Mit Blick auf die Forschungsfragen kann zusammenfassend festgestellt werden, dass sich, trotz des geringen Altersunterschiedes der untersuchten Kinder, alters- und geschlechtsspezifische Unterschiede in den Zeichnungen finden lassen. Ab dem Alter von ca. 12 Jahren erkennen wir Differenzen in der Anzahl der gezeichneten Peergruppe, der Darstellung der Personen und der situativen Rahmung. Die Jungen zeichnen durchgehend eine Gruppe von 
mindestens 10 Mitgliedern. Erst in der Zeichnung des ältesten Jungen (12,7) finden wir körperhafte Darstellungsformen. Es entsteht der Eindruck, dass in den dargestellten Szenen alle gleichermaßen in den Blick genommen werden, nicht der Einzelne wichtig ist, sondern das möglichst abenteuerliche und phantasiereiche Zusammensein. Bei Schuster (1993, S. 39) finden wir den Hinweis, dass das bildnerische Gestalten von Jungen in der ausgehenden Kindheit als „weiblich“ erlebt wird. Die betont abenteuerlichen, wild anmutenden Inhalte der Jungen könnten aus dieser Perspektive als Abgrenzen vom Weiblichen und Betonen des männlichen Habitus gedeutet werden. Der „Fokus auf geteilte Aktivitäten in Freundschaftsbeziehungen ist vor allem als Hinweis auf das Wirksamwerden des maskulinen Stereotyps zu sehen“ (Seiffge-Krenke/Seiffge 2005, S. 271f.).

Den Strichmännchen der Jungen stehen die körperhaften und detaillierten Darstellungen der Mädchen gegenüber. Der Blick der Mädchen auf weniger und ausschließlich weibliche Personen kann auf die zunehmende Bedeutung der Freundinnengruppe in diesem Alter verweisen. Die Einzelnen, die engen Freundinnen, scheinen wichtig zu werden. Die enge Vertrautheit der Mädchen wird über die detailreiche und differenzierte Gestaltung erkennbar (vgl. Breitenbach 2000).

Der veränderte „Strich“, die körperhafte Darstellung in den Zeichnungen der älteren Mädchen und des ältesten Jungen leiten über zur zweiten Forschungsfrage. Die ikonologische Interpretation lie $\beta$ in den Zeichnungen zweier Mädchen und eines Jungen eine Auseinandersetzung mit dem „ZumGeschlecht-Werden“ erkennen. Nachfolgend möchte ich auf zwei dieser Bilder - eines Mädchens und eines Jungen - eingehen.

\subsection{Von Geschlechtssymbolen und dem Fußballspiel zwischen Kaktussen - „Ich bin nur eins von Zweien, oder doch beides?““}

Vom Mädchen zur Frau, vom Jungen zum Mann - lässt sich dieses Entwicklungsthema in den Zeichnungen der Klasse finden?

„Chaos“ - das ist es, was im Bild von M1w $(11,5)$ als erstes in den Blick gerät. 


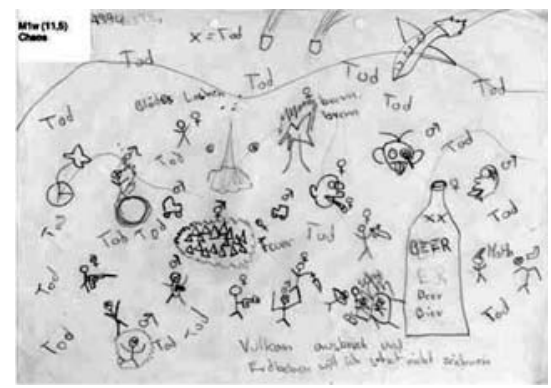

Abb. 2: M1w $(11,5)$ Chaos

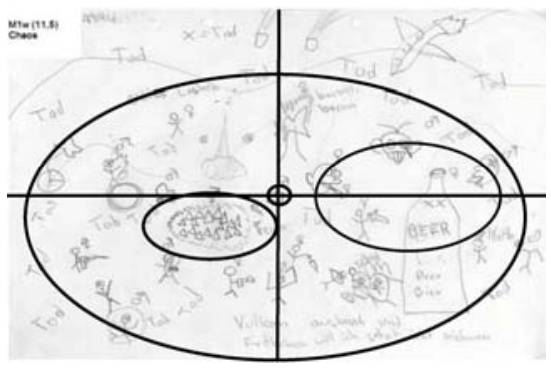

Abb. 3: M1w $(11,5)$ Chaos Planimetrie

Die abgebildeten 17 Personen - 8 männlichen, 6 weiblichen, drei undefinierbaren Geschlechts - sind in einen Kampf verwickelt, der mit Waffen, Raketen und Bomben ausgetragen wird. Die textliche Erläuterung macht uns klar, dass es sich um einen Kampf um Leben und Tod handelt. Die durchkreuzten Figuren sind tot, das vermittelt uns die mathematische Gleichung am oberen Bildrand. Die Dynamik des Geschehens wird durch die Beschriftungen und die vielen zusätzlichen Geschlechtssymbole unterstrichen. Das Zeltlager, zentral in die Gestaltung eingebettet, ist von einem Feuerkranz umgeben. Über die Planimetrie (Abb. 3) und ein in dieser Zeichnung einzigartiges Symbol erhält das Lager einen besonderen Stellenwert. Die Verschmelzung

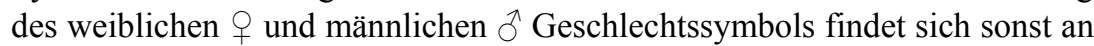
keiner Stelle im Bild. Das Lager ist mit einer welligen Linie eingekreist, die Feuer darstellen soll. Das Feuer breitet sich zwar bedrohlich rund um die 17 (!) Zelte aus, im Lager selbst brennt es allerdings nicht. Der Feuerwall erhält so eine doppelte Bedeutung: auf den ersten Blick und unter dem Eindruck des kämpferischen Geschehens kann er als Gefahr für die Zelte betrachtet werden, er kann aber auch als Schutzwall gesehen werden (vgl. „Übergegensätzlichkeit" bei Imdahl 1996, S. 107).

Bei der Darstellung der Figuren fällt die große räumliche Distanz der Personen auf, deren einzige Kommunikation untereinander das Schießen aufeinander ist. Die Akteurinnen und Akteure sind keine Persönlichkeiten, sie sind nur als Strichfiguren und Träger eines sozialen Identifikationsmerkmals - weiblich oder männlich - dargestellt.

Betrachten wir diese Bilddaten unter dem Aspekt der geschlechtlichen Identifizierung, dann können wir den geschilderten Kampf als einen abstrakten, metaphorischen Kampf im Sinne des Ausbildens einer Geschlechtsidentität interpretieren. Die Bildproduzentin schreibt den Akteur/inn/en Ge- 
schlecht qua Symbol zu. Die Auseinandersetzung mit dieser GeschlechtsZUschreibung findet außerhalb des Lagers statt. Für den Bereich, in dem es keine kämpferischen Auseinandersetzungen, keine Zuschreibungen gibt, gilt das zusammengesetzte Zeichen aus dem weiblichen und männlichen Geschlechtssymbol. Es scheint fast so, als gäbe es dort noch beides - das Männliche und das Weibliche. M1w könnte hier die geschlechtsspezifische Identifizierung am Übergang vom Kind zum Erwachsenen symbolisieren. Über die vielen Geschlechtssymbole, die nicht nur den Strichfiguren sondern auch den Gegenständen zugewiesen werden, könnte sie die zunehmende Sexualisierung der Umwelt thematisieren. M1w gibt an, nicht im Bild zu sein. Könnte sie aber nicht repräsentiert sein durch das Bild? Die Zeichnung würde uns dann einen Blick in ihre innere Gefühlswelt eröffnen. In ihr wäre dann einerseits das Kind, das Aspekte beider Seiten in sich trägt - des Männlichen und des Weiblichen - symbolisiert durch das Lager. Andererseits wäre in ihr aber dann auch ein Kampf mit der Frage: Habe ich als Strichfigur eine weibliche oder männliche Identität? In ihrer Zeichnung sind die weiblichen Strichmännchen siegreich, die männlichen sind „tot“", damit scheint die weibliche Identifizierung zu gewinnen.

Auch das Bild des ältesten Jungen M17m kann als eine Auseinandersetzung mit seinem „Zum-Mann-Werden“ interpretiert werden. Eingebettet in ein Fußballmatch, das zwischen Kaktussen stattfindet, zeichnet er zwei Gruppen: das Fußballteam, das über die körperhafte Darstellung der Spieler und Geschlechtsmerkmale Männlichkeit repräsentiert, und die Gruppe der Klassenkameraden, denen als Strichmännchen auf der Tribüne die Zuschauerrolle zugewiesen wird (Abb. 4). Der Bildproduzent ist Teil beider Gruppen. Er positioniert sich auf halbem Weg zwischen den Strichfiguren und den „Männern“ (Abb. 5). Als Strichmännchen und Mitglied der „Kindergruppe“ ist er nicht gut sichtbar - er hat sich ausradiert. Nur wenn wir genau hinsehen, mit der Lupe, erkennen wir seine Doppelrolle, seine Zwiespältigkeit (Abb. 6). 


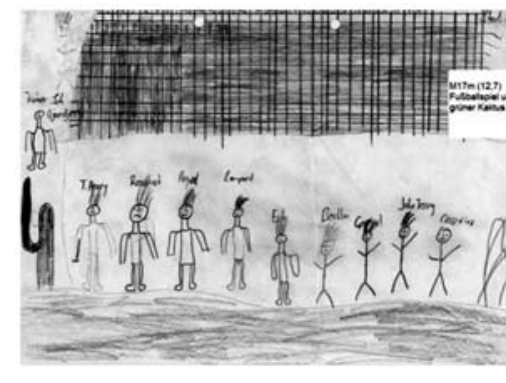

Abb. 4: M17m (12,7) Fußballspiel und grüner Kaktus

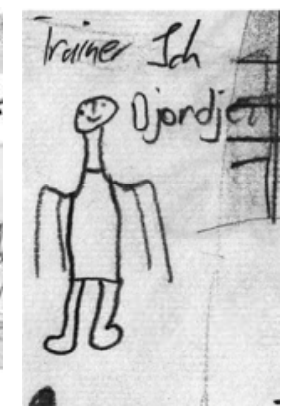

Abb. 5: $\mathrm{M} 17 \mathrm{~m}-$ Am Weg zum Erwachsenen

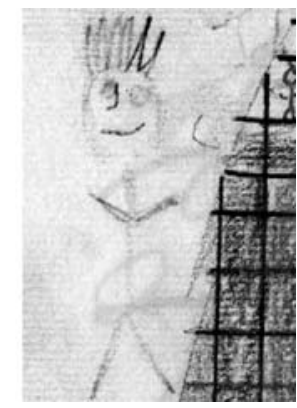

Abb. 6: $\mathrm{M} 17 \mathrm{~m}-$

Kind

Die objektivistische Interpretation der beiden Zeichnungen lässt uns in einem ersten Blick ein kriegerisches Chaos bzw. eine Gruppe von neun Männchen zwischen zwei Kaktussen sehen. In der Analyseeinstellung der dokumentarischen Methode, bei der Frage des WIE, können beide Bilder als eine Auseinandersetzung mit Geschlecht und der eigenen Geschlechtszugehörigkeit betrachtet werden. In ihrer Zeichnung schildert M1w, auf dem Hintergrund der körperlichen Veränderungen und der gesellschaftlichen Erwartungen, den inneren Prozess, zwischen der Vorstellung, das eigene Geschlecht als nur eins von Zweien anzuerkennen und der Vorstellung, beide Geschlechtsmöglichkeiten in sich zu vereinen, hin- und hergerissen zu sein. Im Sinne des intersubjektiven Paradigmas könnte das Lager von M1w mit den Symbolen des Weiblichen und Männlichen als eine Wiederbelebung der bisexuellen Identifizierung der präödipalen Periode gelesen werden (vgl. Benjamin 2002, S. 85f.). Die Tatsache des „Zum-Geschlecht-Werdens“ macht die Unterscheidung zwischen männlich und weiblich bewusst und fordert auf, die eigene Geschlechtsidentität zu konstruieren, sich mit den eigenen Vorstellungen des Frau-Seins, Mann-Seins auseinanderzusetzen. Gehen wir von nebeneinander existierenden Entwicklungspositionen aus, dann zeigen uns die Bilder, dass die Kinder zwischen den beiden Positionen schwanken. Sie sind auf dem Weg zur Frau, zum Mann, gleichzeitig tragen sie aber Vorstellungen, Phantasien in sich, beide Möglichkeiten in sich zu vereinen.

Die dokumentarische Interpretation der Zeichnungen legt nahe, dass die Mädchen und Jungen in der ausgehenden Kindheit vor der Herausforderung stehen, sich als ein einheitliches, beständiges und zusammenhängendes Subjekt zu verstehen. Gleichzeitig scheinen sie nach einer Möglichkeit zu su- 
chen, sich als vielfältig und fragmentarisch, in verschiedenen Kontexten als jeweils anders wahrnehmen zu können. Betrachten wir die Zeichnungen noch einmal im Sinne des Ausbildens einer Geschlechtsidentität, dann kann uns die Analyse der ersten Bilder zur Hypothese veranlassen, dass sie sowohl Geschlechtsidentität (gender identity) als auch Geschlechtsvielfalt (gender multiplicity) brauchen, um sich als Person erleben zu können (vgl. Aron 1995).

Die Analyse der weiteren Zeichnungen wird die sich hier abbildende „Identitätsbaustelle“ verstärkt in den Blick nehmen und nach weiteren Hinweisen suchen, die auf eine Auseinandersetzung mit den körperlichen Veränderungen und der Übernahme einer (Geschlechts-)Identität schließen lassen offen für alters- und/oder geschlechtstypische Differenzen.

\section{Literatur}

Aron, Lewis (1995): Die internalisierte Urszene, in: Benjamin, Jessica (Hrsg.): Unbestimmte Grenzen. Beiträge zur Psychoanalyse der Geschlechter, Frankfurt a.M., S. $19-55$

Benjamin, Jessica (2002): Der Schatten des Anderen. Intersubjektivität Gender Psychoanalyse, Frankfurt a.M. u.a.

Benjamin, Jessica (1996): Die Fesseln der Liebe. Psychoanalyse, Feminismus und das Problem der Macht, Frankfurt a.M.

Bohnsack, Ralf $\left(2007^{6}\right)$ : Rekonstruktive Sozialforschung. Einführung in qualitative Methoden, Opladen

Breitenbach, Eva (2000): Mädchenfreundschaften in der Adoleszenz. Eine fallrekonstruktive Untersuchung in der Gleichaltrigengruppe, Opladen

Butler, Judith (1995): Körper von Gewicht. Die diskursiven Grenzen des Geschlechts, Berlin

Bründel, Heidrun/Hurrelmann, Klaus (1996): Einführung in die Kindheitsforschung, Weinheim und Basel

Dausien, Bettina (2006): Geschlechterverhältnisse und ihre Subjekte, in: Bilden, Helga/Dausien, Bettina (Hrsg.): Sozialisation und Geschlecht. Theoretische und methodologische Aspekte, Opladen, S. 17-44

Hurrelmann, Klaus $\left(1999^{6}\right)$ : Lebensphase Jugend. Eine Einführung in die sozialwissenschaftliche Jugendforschung, Weinheim u.a.

Imdahl, Max $\left(1996^{3}\right)$ : Giotto Arenafresken. Ikonographie Ikonologie Ikonik, München

Jagose, Annamarie (2001): Queer Theory. Eine Einführung, Berlin

Maihofer, Andrea (2004): Geschlecht als hegemonialer Diskurs und gesellschaftlichkulturelle Existenzweise. Neuere Überlegungen zu einer kritischen Theorie von Geschlecht, in: Hartmann, Jutta (Hrsg.): Grenzverwischungen. Vielfältige Lebensweisen im Gender-, Sexualitäts- und Generationendiskurs, Innsbruck, S. 33-40 
Maihofer, Andrea (2002): Geschlecht und Sozialisation, in: Erwägen, Wissen, Ethik, H.1/2002, S. 14-74

Prengel, Annedore (2000): Perspektivitätstheoretische Fragen an die (De-) Konstruktionsdebatte, in: Lemmermöhle, Doris et al.: Lesarten des Geschlechts. Zur DeKonstruktiobsdebatte in der erziehungswissenschaftlichen Geschlechterforschung, Opladen, S. 86-95

Reiß, Wolfgang (2000): Zur Produktion und Analyse von Kinderzeichnungen, in: Heinzel, Friederike (Hrsg.): Methoden der Kindheitsforschung. Ein Überblick über Forschungszugänge zur kindlichen Perspektive, Weinheim, S. 231-244

Rendtorff, Barbara (2006): Zur Bedeutung von Geschlecht im Sozialisationsprozess. Reale, imaginäre und symbolisch-politische Dimensionen des Körpers, in: Bilden, Helga/Dausien, Bettina (Hrsg.): Sozialisation und Geschlecht. Theoretische und methodologische Aspekte, Opladen, S. 89-102

Richter, Hans-Günther (1987): Die Kinderzeichnung. Entwicklung Interpretation Ästhetik, Düsseldorf

Schuster, Martin $\left(1993^{2}\right)$ : Die Psychologie der Kinderzeichnung, Berlin

Seiffge-Krenke, Inge/Seiffge, Jakob Moritz (2005): Die Bedeutung von Freundschaftsbeziehungen, in: King, Vera/Flaake, Karin (Hrsg.): Männliche Adoleszenz. Sozialisation und Bildungsprozesse zwischen Kindheit und Erwachsenensein, Frankfurt a.M., S. 267-286 